\title{
INVERSE BOUNDARY PROBLEMS FOR SYSTEMS IN TWO DIMENSIONS
}

\author{
PIERRE ALBIN, COLIN GUILLARMOU, LEO TZOU, AND GUNTHER UHLMANN
}

\begin{abstract}
We prove identification of coefficients up to gauge by Cauchy data at the boundary for elliptic systems on oriented compact surfaces with boundary or domains of $\mathbb{C}$. In the geometric setting, we fix a Riemann surface with boundary, and consider both a Dirac-type operator plus potential acting on sections of a Clifford bundle and a connection Laplacian plus potential (i.e. Schrödinger Laplacian with external Yang-Mills field) acting on sections of a Hermitian bundle. In either case we show that the Cauchy data determines both the connection and the potential up to a natural gauge transformation: conjugation by an endomorphism of the bundle which is the identity at the boundary. For domains of $\mathbb{C}$, we recover zeroth order terms up to gauge from Cauchy data at the boundary in first order elliptic systems.
\end{abstract}

\section{INTRODUCTION}

In this work, we show that the Cauchy data space at the boundary identifies the coefficients (up to gauge) of a certain type of first order and second order elliptic systems on a Riemann surface and domains of $\mathbb{C}$, generalizing the results of [11]. We show here that Cauchy data at the boundary of a Riemann surface determine:

1) the 0 -th order term (up to gauge invariance) in the operator $D+V$ where $D$ is a Dirac type operator and $V$ an endomorphism on a clifford bundle,

2) the connection $\nabla$ and the potential $V$ (up to gauge) acting on a complex vector bundle in the Schrödinger connection Laplacian $\nabla^{*} \nabla+V$,

\subsection{Connection Laplacians on surfaces, Schrödinger operator with external Yang-} Mills field. Let $M$ be a Riemann surface with boundary and $\pi: E \rightarrow M$ be a complex vector bundle equipped with a Hermitian structure $\langle\cdot, \cdot\rangle_{E}$. We denote the space of $E$-valued $k$-forms by $\Omega^{k}(E)=C^{\infty}\left(M ; \Lambda^{k} T^{*} M \otimes E\right)$ and similarly use $\Omega^{p, q}(E)$ to denote $E$ valued forms of type $(p, q)$. Let $\nabla$ be a connection on $E$ and consider the connection Laplacian $L:=\nabla^{*} \nabla$ where the adjoint $\nabla^{*}$ is taken with respect to the Hermitian inner product. For $V \in L^{\infty}(M, \operatorname{End}(E))$, we define the Cauchy data space of the operator $L+V$ by

$$
\mathcal{C}_{L+V}:=\left\{\left.\left(u, \nabla_{\nu} u\right)\right|_{\partial M} \in H^{\frac{1}{2}}(\partial M, E) \times H^{-\frac{1}{2}}(\partial M, E) ;(L+V) u=0, u \in H^{1}(M, E)\right\}
$$

where $\nu$ is the inward normal vector field to the boundary.

The Cauchy data space can not determine the connection $\nabla$ and the potential $V$, for there is a gauge invariance. Indeed, it suffices to consider the conjugation of $L+V$ by a unitary

P. A. was supported by a postdoctoral fellowship of the Foundation Sciences Mathématiques de Paris.

C.G. is supported by ANR Grants No. ANR-09-JCJC-0099-01 and ANR-10-BLAN 0105.

L.T. was supported by NSF Grant No. DMS-0807502.

G.U. was supported by NSF, a Chancellor Professorship at UC Berkeley and a Senior Clay Award. 
section

$$
F \in C^{\infty}(M ; \operatorname{End}(E)), \quad F^{*}=F^{-1},\left.\quad F\right|_{\partial M}=\mathrm{Id} .
$$

There is a natural lift of $F$ to a unitary endomorphism in $C^{\infty}\left(M ; \operatorname{End}\left(T^{*} M \otimes E\right)\right)$, still denoted $F$, defined by $(F \sigma)(X):=F(\sigma(X))$ for all $\sigma \in C^{\infty}\left(M ; T^{*} M \otimes E\right)$ and $X \in T M$, and it is easy to see that the Cauchy data space of $\widetilde{L}+\widetilde{V}=\left(F^{-1} \nabla F\right)^{*}\left(F^{-1} \nabla F\right)+F^{-1} V F$ is the same as the Cauchy data space of $L+V$,

$$
\mathcal{C}_{\widetilde{L}+\widetilde{V}}=\mathcal{C}_{L+V}
$$

In this paper, we prove that the Cauchy data space determines $\nabla$ and $V$ up to gauge. Before we state the result, we use the notation $C^{r}(M)$ (with $r \geq 0$ ) for the usual $r$-Hölder space on $M$ and $W^{s, p}(M)$ (with $p \in[1, \infty], s \in \mathbb{R}$ ) for the Sobolev space with $s$ derivatives in $L^{p}(M)$, while $H^{s}(M):=W^{2, p}(M)$.

A connection on a vector bundle $E$ is said to be in $C^{r}$ (or similarly $W^{s, p}$ ) if its (local) connection form is in $C^{r}\left(M ; \operatorname{End}(E) \otimes T^{*} M\right)$.

Theorem 1. Let $\nabla_{1}$ and $\nabla_{2}$ be two Hermitian connections on a smooth Hermitian bundle $E$, of complex dimension $n$ and let $V_{1}, V_{2}$ be two sections of the bundle $\operatorname{End}(E)$. We assume that $\nabla_{j}$ have the regularity $C^{r} \cap W^{s, p}(M)$ with

$$
0<r<s, \quad p \in(1, \infty) \text { satisfy } r+s>1, \quad r \notin \mathbb{N}, \quad s p>2 n+2
$$

and that $V_{j} \in W^{1, q}(M)$ with $q>2$. Let $L_{j}:=\nabla_{j}^{*} \nabla_{j}$ and assume that the Cauchy data spaces agree $\mathcal{C}_{L_{1}+V_{1}}=\mathcal{C}_{L_{2}+V_{2}}$, then there exists a unitary endomorphism $F \in C^{1}(M ; \operatorname{End}(E))$, satisfying $\left.F\right|_{\partial M}=\mathrm{Id}$, such that $\nabla_{1}=F^{-1} \nabla_{2} F$ and $V_{1}=F^{-1} V_{2} F$.

Observe that Theorem 1 is a generalization of the scalar trivial bundle case in [11] where $E=M \times \mathbb{C}$ and $\nabla_{j}=d+i X_{j}$ for $X_{j}$ a real valued 1-form. For scalar trivial bundle on domains of $\mathbb{C}$, this was first proved (with partial data measurement) by Imanuvilov-UhlmannYamamoto [18, 19]. Our result is new even in the case of domains in $\mathbb{C}$ when the bundle is not a line bundle (this was known only under smallness assumption, see Li [24]).

We prove Theorem 1 as a consequence of an identifiability result for Dirac-type systems.

0.2. Dirac systems on surfaces. A Dirac vector bundle (also known as a Clifford vector bundle) on a Riemannian manifold $(M, g)$ is a complex vector bundle $E \longrightarrow M$ together with a Clifford multiplication map,

$$
\gamma: C^{\infty}\left(M ; T^{*} M\right) \longrightarrow C^{\infty}(M ; \operatorname{End}(E)), \quad \text { s.t. } \gamma(\eta) \gamma(\omega)+\gamma(\omega) \gamma(\eta)=-2 g(\eta, \omega),
$$

a Hermitian metric $\langle\cdot, \cdot\rangle_{E}$ and a Hermitian connection $\nabla$ satisfying

$$
\langle\gamma(\omega) s, t\rangle_{E}=-\langle s, \gamma(\omega) t\rangle_{E}, \quad\left[\nabla_{W}, \gamma(\omega)\right]=\gamma\left(\nabla_{W} \omega\right)
$$

for every $\omega \in C^{\infty}\left(M ; T^{*} M\right), s, t \in C^{\infty}(M, E)$, and $W \in C^{\infty}(M ; T M)$. In dimension 2 , there is a chirality operator defined by

$$
H:=i \gamma\left(\theta^{1}\right) \gamma\left(\theta^{2}\right)
$$

where $\left(\theta^{1}, \theta^{2}\right)$ is any local orthonormal basis of $T^{*} M$. One easily checks that $H$ does not depend on the choice of $\left(\theta^{1}, \theta^{2}\right)$ and therefore it can be defined globally on the surface. Since $H^{2}=\mathrm{Id}$, it determines a splitting of $E$,

$$
E=E^{+} \oplus E^{-},
$$


the connection preserves this splitting and Clifford multiplication reverses it. The Dirac-type operator associated to this data is the composition

$$
D: H^{1}(M ; E) \stackrel{\nabla}{\rightarrow} L^{2}\left(M ; T^{*} M \otimes E\right) \stackrel{\gamma}{\rightarrow} L^{2}(M ; E) .
$$

It is self-adjoint with respect to $\langle\cdot, \cdot\rangle_{E}$, and odd with respect to the splitting of $E$. As above, if $V \in W^{1, p}(M, \operatorname{End}(E))$, we can define the Cauchy data space of $D+V$ by

$$
\mathcal{C}_{D+V}:=\left\{\left.u\right|_{\partial M} \in H^{\frac{1}{2}}(\partial M, E) ;(D+V) u=0, u \in H^{1}(M, E)\right\} .
$$

We then prove the following theorem:

Theorem 2. Let $\left(E,\langle\cdot, \cdot\rangle, \gamma, \nabla_{j}\right), j=1,2$ be two Dirac bundles on a Riemann surface $M$ with boundary. We assume the bundle and $\gamma$ are smooth, while $\nabla_{j}$ is $C^{r} \cap W^{s, p}$ with $s, p, r$ as in (2). Let $V_{1}, V_{2}$ be $W^{1, q}$ sections of $\operatorname{End}(E)$, with $q>2$. Suppose that the Cauchy data spaces $\mathcal{C}_{D_{1}+V_{1}}$ and $\mathcal{C}_{D_{2}+V_{2}}$ coincide, then there exist $C^{1}$ bundle-morphisms $\Phi, \Psi: E \rightarrow E$, preserving the splitting $E=E^{+} \oplus E^{-}$, with $\Psi=\Phi=\mathrm{Id}$ on $\partial M$ and such that $\Phi\left(D_{1}+V_{1}\right) \Psi=D_{2}+V_{2}$.

As we shall prove, this theorem actually follows from the particular case:

Proposition 3. Let $\underline{\mathbb{C}}^{n}:=M \times \mathbb{C}^{n}$ and $E:=\underline{\mathbb{C}}^{n} \oplus\left(\underline{\mathbb{C}}^{n} \otimes\left(T^{0,1} M\right)^{*}\right)$. Consider the operators $D+V_{j}, j=1,2$, defined by $D:=\left(\begin{array}{cc}0 & \bar{\partial}^{*} \\ \bar{\partial} & 0\end{array}\right), V_{j}=\left(\begin{array}{cc}Q_{j}^{+} & A_{j} \\ B_{j} & Q_{j}^{-}\end{array}\right)$, acting on sections of $E$, with $A, B \in W^{1, q}(M), q>2$, and $Q^{ \pm} \in C^{r} \cap W^{s, p}(M)$ such that $r, s, p$ satisfies the condition (2.2). If the Cauchy data spaces $\mathcal{C}_{D+V_{1}}$ and $\mathcal{C}_{D+V_{2}}$ agree, then there exist $C^{1}$ bundle isomorphisms $F, G$ of $\underline{\mathbb{C}}^{n}$ such that $\left.F\right|_{\partial M}=\left.G\right|_{\partial M}=\mathrm{Id}$ and, as operators,

$$
D+V_{2}=\left(\begin{array}{cc}
G & 0 \\
0 & F^{-1}
\end{array}\right)\left(D+V_{1}\right)\left(\begin{array}{cc}
F & 0 \\
0 & G^{-1}
\end{array}\right) .
$$

The proof of this identification result is based on previous work [11] of the second and third authors, itself based on a new idea of Bughkeim [7], and the construction of holomorphic phases on Riemann surfaces in [9].

0.3. Systems in domains of $\mathbb{C}$. For domains $\Omega$ of $\mathbb{C}$, our proof shows the identification up to gauge of zeroth order terms $V$ by Cauchy data for any elliptic $(m+n) \times(m+n)$ systems of the form

$$
\left(\begin{array}{ll}
\bar{\partial} & 0 \\
0 & \partial
\end{array}\right)\left(\begin{array}{l}
u \\
v
\end{array}\right)+\left(\begin{array}{cc}
A & Q^{+} \\
Q^{-} & B
\end{array}\right)\left(\begin{array}{l}
u \\
v
\end{array}\right)=0
$$

where $\bar{\partial}$ acts on each component of $\mathbb{C}^{m}$ valued functions $u$ by $\partial_{\bar{z}}$ and $\partial$ acts on each component of $\mathbb{C}^{n}$ valued functions $v$ by $\partial_{z}$, and $Q^{ \pm}, A, B$ are matrix valued functions satisfying similar asumptions as in Proposition 3. We refer to Theorem 4.1 below for a precise statement.

0.4. State of the art in two dimensions. Let us recall some known results about Calderón inverse type problem in dimension 2 (we do not discuss here references of higher dimensional results).

For inverse problems on domains in $\mathbb{C}$, Nachman [26] proved that the Cauchy data space determines a $C^{2}$-conductivity (with a reconstruction method). Before that, Sylvester [30] showed how to reduce the problem for anisotropic conductivities to isotropic conductivities.

Brown and Uhlmann [6] used the $\bar{\partial}$ factorization of Beals-Coifman [3] for solving the identification result for the isotropic conductivity with regularity $W^{1, p}$ for $p>2$. The most 
general result in terms of regularity is for $L^{\infty}$-conductivity by Astala-Päivärinta [1] (and [2] for anisotropic case), using quasiconformal methods. The identification of an $L^{\infty}$-potential in the Schrödinger operator in a domain of $\mathbb{C}$ was proved recently by Bukhgeim [7, after the problem had been open for more than 20 years. This was then extended with only partial data measurements for domains in $\mathbb{C}$ by Imanuvilov-Uhlmann-Yamamoto [17. For magnetic Schrödinger operators, Kang-Uhlmann [21] showed identification of a magnetic field (up to gauge) and a potential in a simply connected domain under smallness assumption in $L^{\infty}$ norm of the potential. For general elliptic second operators on domains in $\mathbb{C}$, and with only partial data measurements, the identification was shown by Imanuvilov-Uhlmann-Yamamoto [18, 19, 20, while identification for partial data with disjoints measurements for Dirichlet and Neumann data has been proved by the same authors in [20].

For what concerns elliptic systems, Novikov-Santacesaria [28] considered recently $\Delta+V$ acting on vector valued functions on a domain of $\mathbb{C}$, with $V$ a metric potential, they show identification of $V$ from Cauchy data space. Li [24] proved identifiability up to gauge for Yang-Mills Schrödinger system under smallness assumption on the coefficients.

In the geometric case, the determination of the conformal class of a metric $g$ from the Cauchy data space of $\Delta_{g}$ on a Riemann surface with boundary was first shown by LassasUhlmann [23, followed by Belishev [4] and Henkin-Michel [12] (with a reconstruction procedure). The identification of an isotropic conductivity on a Riemann surface was shown by Henkin-Michel [13] with reconstruction; it was extended by Henkin-Santacesaria [15] to anisotropic conductivities for surfaces embedded in $\mathbb{R}^{3}$. Guillarmou-Tzou [9, 10] show identification of a $C^{1, \alpha}$-potential in the Schrödinger potential on a fixed Riemann surface (with only partial measurement), generalizing the result of [17] to Riemann surfaces. In [11], it is proved that one can determine a connection (up to gauge) and a potential in the connection Laplacian with potential on a complex line bundle (from the full Cauchy data space).

The paper is organized as follows: we first prove that a Dirac type system on a Riemann surface with boundary can be reduced to a $\bar{\partial}, \bar{\partial}^{*}$ type system on the trivial bundle $M \times \mathbb{C}^{2 n}$. Then we prove Theorem 2 by using the Complex Geometric Optics method (also called Faddeev exponential solutions) developed by Bughkeim [7 for domains in $\mathbb{C}$, and extended by 11 to Riemann surfaces. Finally we show that the inverse problem for the connection Laplacian of Theorem 1 can be reduced to Theorem 2 .

\section{DiRAC OPERATORS AND $\bar{\partial}$ OPERATORS}

In this section, we show that any system of Dirac type $(D+V) u=0$ on a Dirac vector bundle can be reduced to a $\bar{\partial}, \bar{\partial}^{*}$ system. We will work with holomorphic structures of low regularity following [16].

If $\mathcal{B}$ is a regularity space (such as a Sobolev space or a Hölder space), a holomorphic structure with regularity $\mathcal{B}$ on a smooth complex vector bundle $E$ of rank $n$ over a Riemann surface $M$ with boundary is an atlas of local trivializations $h_{i}:\left.E\right|_{U_{i}} \rightarrow U_{i} \times \mathbb{C}^{n}, i \in I$, with regularity $\mathcal{B}$ such that the transition functions $h_{i j}: U_{i} \cap U_{j} \rightarrow G L(N, \mathbb{C})$ are holomorphic with respect to the holomorphic structure on $M$. A holomorphic section $s: U \rightarrow E$ is a section such that $h_{i} \circ s$ is holomorphic as a map from $U$ to $\mathbb{C}^{n}$. 
Following Hill and Taylor [16, we will usually ask that our holomorphic structures have regularity $C^{r+1} \cap W^{s+1, p}$ where

$$
0<r<s, \quad p \in(1, \infty) \text { satisfy } r+s>1, \quad r \notin \mathbb{N}, \quad s p>2 n+2 .
$$

A holomorphic vector bundle over a surface with boundary is holomorphically trivial. This is shown for instance in Forster [8, Th 30.1 and Th 30.4] for smooth holomorphic structures, and with small modification yields:

Proposition 1.1. Let $E \rightarrow M$ be a holomorphic vector bundle of rank $n$ with regularity $C^{r} \cap W^{s, p}$ over a compact Riemann surface $M$ with non-empty boundary, where $r, s, p$ are as in (1.1). Then $E$ is holomorphically trivial in the sense that there exist $n$ holomorphic sections $f_{1}, \ldots, f_{n} \in C^{r+1} \cap W^{s+1, p}(M, E)$ such that at every point $x \in M, f_{1}(x), \ldots, f_{n}(x)$ are linearly independent in the fiber $E_{x}$.

Closely associated to a holomorphic structure on a complex vector bundle $E \rightarrow M$ is a Cauchy-Riemann type operator. We say that a first order differential operator

$$
P: H^{1}(M ; E) \longrightarrow L^{2}\left(M ;\left(T^{0,1} M\right)^{*} \otimes E\right)
$$

is a $C R$ operator if it satisfies

$$
P(f \xi)=f P(\xi)+(\bar{\partial} f) \xi, \quad \text { for every } f \in C^{\infty}(M), \xi \in C^{\infty}(M, E) .
$$

A CR operator $P$ can be extended to forms and satisfies $P^{2}=0$ since on surfaces there are no $(0,2)$-forms. If a Hermitian product is given on $E$, a $\mathrm{CR}$ operator $P$ is induced by (and induces) a unique Hermitian connection $\nabla$ in the sense that

$$
P \xi=(\nabla \xi)^{0,1}
$$

and we will denote this operator by $\bar{\partial}^{\nabla}$. We say that a holomorphic structure on $E$ is compatible with a CR operator $\bar{\partial} \nabla$ if the holomorphic sections of $E$ are in ker $\bar{\partial}^{\nabla}$.

Given a regularity space $\mathcal{B}$, we say that $\bar{\partial}^{\nabla}$ is a Cauchy-Riemann operator of class $\mathcal{B}$ if there is a holomorphic atlas on $M$ in which $\bar{\partial}^{\nabla}$ can be written locally as

$$
\bar{\partial}^{\nabla}=\bar{\partial}+A
$$

with $A \in \mathcal{B}\left(M, \operatorname{End}(E) \otimes\left(T^{0,1} M\right)^{*}\right)$.

Lemma 1.2. Let $E \rightarrow M$ be a smooth complex vector bundle of rank $n$ and let $r, s, p$ be as in (1.1). There is a $C^{r+1} \cap W^{s+1, p}$ holomorphic structure on $E$ if and only if $E$ can be equipped with a $C R$ operator $P$ of class $C^{r} \cap W^{s, p}$.

Proof. Given a $C^{r+1} \cap W^{s+1, p}$ holomorphic structure on $E$, let

$$
F=\left(f_{1}, \ldots, f_{n}\right): E \rightarrow M \times \mathbb{C}^{n}
$$

where $f_{1}, \ldots, f_{n}$ are the holomorphic sections from Proposition 1.1. Then $F$ is a bundle isomorphism of class $C^{1+r} \cap W^{s+1, p}$. If $\bar{\partial}$ is the standard Cauchy-Riemann operator on the trivial bundle $M \times \mathbb{C}^{n}$ induced by the $\bar{\partial}$ operator on $C^{\infty}(M)$, then $P=F \bar{\partial} F^{-1}$ is a $\mathrm{CR}$ operator on $E$ of class $C^{r} \cap W^{s, p}$ compatible with the holomorphic structure on $E$.

Conversely, given $P$ a CR operator of class $C^{r} \cap W^{s, p}$, we can find a holomorphic structure on $E$ following the proof of Kobayashi [22, Ch. 1, Prop 3.7]. This proof is in the smooth category, but we can modify it slightly to have it in the range of regularity assumed above, by using a low regularity version of the famous Newlander-Nirenberg integrability result [27] due to Hill-Taylor [16]. To construct the holomorphic local trivializations, Kobayashi defines 
an almost-complex structure $J$ on $E$ which is integrable, and with $J$ having the regularity of $A$ when we write the Cauchy-Riemann operator as $\bar{\partial}+A$ in a smooth trivialization $M \times \mathbb{C}^{n}$ of $E$. Consider the same $J$ as Kobayashi (in the proof of Prop 3.7 of [22]), it is $C^{r} \cap W^{s, p}$ since $A \in C^{r} \cap W^{s, p}$ by assumption and $J$ is formally integrable by the argument of Kobayashi (which just comes from $P^{2}=0$ ). We can then use the main result of Hill-Taylor [16] which says that an integrable complex structure $J$ which is $C^{r} \cap W^{s, p}$ on a complex manifold of dimension $n+1$ induces local holomorphic trivializations of $E$ of class $C^{1+r} \cap W^{s+1, p}$ with the $s, r, p$ satisfying the conditions above.

We now show that on a surface with boundary, any Dirac-type operator (4) on a holomorphic vector bundle is induced by a CR-operator.

Lemma 1.3. Let $(E,\langle\cdot, \cdot\rangle, \gamma, \nabla)$ be a Dirac vector bundle of complex rank $2 n$ over a smooth Riemann surface $M$ with boundary, and assume that $\nabla$ and $\gamma$ have the regularity $C^{r} \cap W^{s, p}$ with $r, s, p$ satisfying (1.1). Let $D$ be the associated Dirac operator. Then there exists a complex subbundle $E_{0}$ of $E$ of complex rank $n$ and a bundle isomorphism

$$
B: E \longrightarrow E_{0} \oplus\left(E_{0} \otimes\left(T^{(0,1)} M\right)^{*}\right)
$$

such that

$$
B D B^{-1}=\sqrt{2}\left(\bar{\partial}^{\nabla}+\left(\bar{\partial}^{\nabla}\right)^{*}\right)
$$

where $\bar{\partial} \nabla$ is the CR operator associated with the Hermitian connection $\nabla$ on $E$.

Proof. Endow $E$ with the holomorphic structure induced by the Hermitian connection $\nabla$ through its $\bar{\partial}^{\nabla}$, this is a $C^{1+r} \cap W^{1+s, p}$ holomorphic structure. Both $T^{1,0} M$ and $E$ are trivial holomorphic bundles by Proposition 1.1 .

Choose (pointwise orthonormal) sections $Z \in C^{\infty}\left(M ; T^{1,0} M\right), \bar{Z} \in C^{\infty}\left(M ; T^{0,1} M\right)$ and let $S, T$ be real sections in $C^{\infty}(M ; T M)$ such that $T=J S$, where $J \in \operatorname{End}(T M)$ is the complex structure on $T M$, and

$$
\bar{Z}=\frac{1}{2}(S+i T)
$$

(note that $|S|=|T|=\sqrt{2}$ ). After extending $\gamma$ to be $\mathbb{C}$-linear and defining $S^{*}, T^{*}$ the dual basis to $S, T$ and $Z^{*}:=S^{*}+i T^{*}, \bar{Z}^{*}:=S^{*}-i T^{*}$ we can write the Dirac-type operator as

$$
D=\gamma\left(S^{*}\right) \nabla_{S}+\gamma\left(T^{*}\right) \nabla_{T}=\gamma\left(Z^{*}\right) \nabla_{Z}+\gamma\left(\bar{Z}^{*}\right) \nabla_{\bar{Z}}
$$

Notice that

$$
\gamma\left(Z^{*}\right)^{2}=\gamma\left(\bar{Z}^{*}\right)^{2}=0 \quad \text { and } \quad \gamma\left(Z^{*}\right) \gamma\left(\bar{Z}^{*}\right)+\gamma\left(\bar{Z}^{*}\right) \gamma\left(Z^{*}\right)=-2,
$$

and hence the image of $\gamma\left(Z^{*}\right)$ is equal to its null space, and $\gamma\left(\bar{Z}^{*}\right)$ establishes an isomorphism between the image of $\gamma\left(Z^{*}\right)$ and its orthogonal complement (recall that $\gamma\left(\bar{Z}^{*}\right)^{*}=-\gamma\left(Z^{*}\right)$ by (3) ). That is, we have

$$
E=E_{0} \oplus \gamma\left(\bar{Z}^{*}\right) E_{0}, \text { with } E_{0}=\gamma\left(Z^{*}\right) E .
$$

The bundle $E_{0}$ is a complex subbundle of rank $n$ of $E$, and is trivial on $M$. We can then define

$$
\begin{aligned}
B: E=E_{0} \oplus \gamma\left(\bar{Z}^{*}\right) E_{0} & \longrightarrow E_{0} \oplus\left(\left(T^{0,1} M\right)^{*} \otimes E_{0}\right), \\
v+\gamma\left(\bar{Z}^{*}\right) w & \longmapsto w+\sqrt{2} v \bar{Z}^{*}
\end{aligned}
$$

and from (1.2) we see that

$$
B\left(\gamma\left(\bar{Z}^{*}\right) \cdot\right)=\sqrt{2} \mathfrak{e}\left(\bar{Z}^{*}\right) B(\cdot), \quad B\left(\gamma\left(Z^{*}\right) \cdot\right)=-\sqrt{2} \mathfrak{i}\left(\bar{Z}^{*}\right) B(\cdot)
$$


where $\mathfrak{e}$ denotes the exterior product and $\mathfrak{i}$ denotes the interior product. Since we also have [25, Remark C.1.3] $\left(\bar{\partial}^{\nabla}\right)^{*}=-i * \partial^{\nabla}=-\mathfrak{i}\left(\bar{Z}^{*}\right) \nabla_{Z}$, this allows us to identify

$$
B(D \cdot)=\sqrt{2}\left(\bar{Z}^{*} \wedge \nabla_{\bar{Z}}-\mathfrak{i}\left(\bar{Z}^{*}\right) \nabla_{Z}\right) B(\cdot)=\sqrt{2}\left(\bar{\partial}^{\nabla}+\left(\bar{\partial}^{\nabla}\right)^{*}\right) B(\cdot) .
$$

as required.

It will be useful to recall the Lichnerowicz formula of Bochner-Kodaira for the Dirac-type operator associated to the CR-operator, $\sqrt{2}\left(\bar{\partial}^{\nabla}+\left(\bar{\partial}^{\nabla}\right)^{*}\right)$, namely [5, Proposition 3.71]

$$
\bar{\partial}^{\nabla}\left(\bar{\partial}^{\nabla}\right)^{*}+\left(\bar{\partial}^{\nabla}\right)^{*} \bar{\partial}^{\nabla}=\nabla^{*} \nabla+\sum_{j, k} \mathfrak{e}\left(d \bar{z}^{j}\right) \mathfrak{i}\left(d z^{k}\right) F^{E_{0} \otimes K^{*}}\left(\partial_{z_{k}}, \partial_{\bar{z}_{j}}\right)=\nabla^{*} \nabla+\Omega_{\Lambda^{*}\left(T^{0,1} M\right)^{*} \otimes E_{0}} .
$$

where $F^{E_{0} \otimes K^{*}}$ is the twisting curvature of the connection, which here reduces to the curvature 2-form, $\Omega_{\Lambda^{*}\left(T^{0,1} M\right)^{*} \otimes E_{0}}$. The latter equality holds as we are working on a surface, which also means that

$$
\bar{\partial}^{\nabla}\left(\bar{\partial}^{\nabla}\right)^{*}+\left(\bar{\partial}^{\nabla}\right)^{*} \bar{\partial}^{\nabla}= \begin{cases}\left(\bar{\partial}^{\nabla}\right)^{*} \bar{\partial}^{\nabla} & \text { on } E_{0} \\ \bar{\partial}^{\nabla}\left(\bar{\partial}^{\nabla}\right)^{*} & \text { on } E_{0} \otimes T_{0,1}^{*} M\end{cases}
$$

Since $E \rightarrow M$ is holomorphically trivial, Proposition 1.1 gives us a bundle isomorphism $F=\left(f_{1}, \ldots, f_{2 n}\right): E \rightarrow M \times \mathbb{C}^{2 n}$ such that $F \bar{\partial} \nabla=\bar{\partial} F$. Taking adjoints,

$$
\left(\bar{\partial}^{\nabla}\right)^{*} F^{*}=F^{*}(\bar{\partial})^{*}
$$

and hence the Bochner-Kodaira formula yields

$$
\nabla^{*} \nabla=-\left(\bar{\partial}^{\nabla}\right)^{*} \bar{\partial}^{\nabla}-\Omega=-F^{*}(\bar{\partial})^{*}\left(F^{*}\right)^{-1} F^{-1} \bar{\partial} F-\Omega
$$

over $E_{0}$ and a similar formula over $\left(T^{0,1} M\right)^{*} \otimes E_{0}$. This factorization formula will be useful for our considerations below.

\section{IDENTIFICATION FOR DiRAC SYSTEMS}

In this section we will prove Theorem 2: the Cauchy data of a Dirac operator plus potential on a surface with boundary identifies the operator up to a unitary endomorphism equal to the identity at the boundary.

In the previous section, the bundle $E_{0}$ is a complex subbundle of rank $n$ on $M$, it is then trivial and can be identified with $\underline{\mathbb{C}}^{n}:=M \times \mathbb{C}^{n}$. Moreover, by Lemma 1.3, we may assume that the Dirac bundle is a $\bar{\partial}$ system on a trivial $\mathbb{C}^{2 n}$ bundle, so in this section we will study operators of the form

$$
D+V:=\left(\begin{array}{cc}
0 & \bar{\partial}^{*} \\
\bar{\partial} & 0
\end{array}\right)+\left(\begin{array}{cc}
Q^{+} & A^{*} \\
A & Q^{-}
\end{array}\right)
$$

acting as a bounded operator from $H^{1}(M, E)$ to $L^{2}(M, E)$ where $E:=\underline{\mathbb{C}}^{n} \oplus\left(\underline{\mathbb{C}}^{n} \otimes\left(T^{0,1} M\right)^{*}\right)$,

$$
\begin{gathered}
A, A^{\prime} \in C^{r} \cap W^{s, p}\left(M, \operatorname{End}\left(\mathbb{C}^{n}\right) \otimes\left(T^{0,1} M\right)^{*}\right), \text { with } r, s, p \text { as in (1.1), } \\
\text { and } Q^{ \pm} \in W^{1, q}(M), q>2 .
\end{gathered}
$$

Notice that by Sobolev embedding, $Q^{ \pm} \in L^{\infty}(M)$.

First consider the case where $A=A^{\prime}=0$, so that we want to determine $Q^{ \pm}$from the Cauchy data. The proof of [11, Lemma 7.4] applies verbatim to a matrix valued potential and shows that

$$
\left.Q^{ \pm}\right|_{\partial M} \text { are determined by } \mathcal{C}_{D+V} ;
$$


in 2.2 we will show that in fact $\mathcal{C}_{D+V}$ determines $Q^{ \pm}$on all of $M$. We do this, following the arguments in [7] and [11], by showing that for a dense set of points $z \in M$ one can find a solution of 'complex geometric optics' (or CGO) type that determines the potential at that point. These solutions are constructed in $\$ 2.1$ and then in $\$ 2.3$ we show that we can reduce the general case to the case $A=A^{\prime}=0$.

2.1. Solutions from complex geometric optics. In this section, we use the method of [11, based on the work of Bughkeim [7] to construct elements in the null space of $D+V$ of CGO-type (where $V$ is as in (2.2) with $A=A^{\prime}=0$ ). These solutions will have Morse holomorphic or anti-holomorphic phases, so we start by recalling the following Proposition, proved in [9, Prop 2.1]:

Proposition 2.1. On a Riemann surface with boundary, there exists a dense set of points $p$ in $M$ such that there exists a holomorphic function $\Phi$ which is Morse, i.e. $d \Phi$ has only zeros of order 1, and with a critical point at $p$.

Let $\Phi$ be a holomorphic Morse function and $h>0$ a small parameter. For certain choices of

$$
a \in C^{\infty}\left(M ; \underline{\mathbb{C}}^{n}\right), \quad \bar{\partial} a=0, \quad b \in C^{\infty}\left(M ; T_{0,1}^{*} M \otimes \underline{\mathbb{C}}^{n}\right), \quad \bar{\partial}^{*} b=0
$$

we will show that one can find $r_{h}, s_{h}$ with small $L^{p}$ norms as $h \rightarrow 0$ so that

$$
U_{h}=\left(\begin{array}{l}
e^{\Phi / h}\left(a+r_{h}\right) \\
e^{\bar{\Phi} / h}\left(b+s_{h}\right)
\end{array}\right)
$$

is in the null space of $D+V$.

Since $\bar{\partial} \Phi=0$ and $\bar{\partial}^{*} \bar{\Phi}=0$, solving $(D+V) U_{h}=0$ for $r_{h}, s_{h}$ is equivalent to

$$
\left(D+V_{\psi}\right)\left(\begin{array}{l}
a+r_{h} \\
b+s_{h}
\end{array}\right)=\left(\begin{array}{l}
0 \\
0
\end{array}\right)
$$

where $\psi:=\operatorname{Im}(\Phi)$ and

$$
V_{\psi}:=\left(\begin{array}{cc}
e^{-\bar{\Phi} / h} & 0 \\
0 & e^{-\Phi / h}
\end{array}\right) V\left(\begin{array}{cc}
e^{\Phi / h} & 0 \\
0 & e^{\bar{\Phi} / h}
\end{array}\right)=\left(\begin{array}{cc}
e^{\frac{2 i \psi}{h}} Q^{+} & 0 \\
0 & e^{-\frac{2 i \psi}{h}} Q^{-}
\end{array}\right),
$$

and hence to

$$
\left(D+V_{\psi}\right)\left(\begin{array}{l}
r_{h} \\
s_{h}
\end{array}\right)=-V_{\psi}\left(\begin{array}{l}
a \\
b
\end{array}\right) .
$$

Next we make use of a right inverse for $D$ as constructed in Proposition 2.1 and Lemma 2.1 of the paper [11].

Proposition 2.2. There exists an operator $D^{-1}: L^{q}(M, E) \rightarrow W^{1, q}(M, E)$, bounded for all $q \in(1, \infty)$, such that $D D^{-1}=\mathrm{Id}$.

Proof. The proof in 11 is written for the case of a line bundle, i.e. $n=1$, but since here the bundle is the trivial $\mathbb{C}^{n}$ bundle, the operator $D$ splits as a direct sum of operators on complex line bundles and the proof is then contained in [11]. The operator $D^{-1}$ is of the form

$$
D^{-1}=\left(\begin{array}{cc}
0 & \mathcal{R} \bar{\partial}^{-1} \mathcal{E} \\
\mathcal{R}\left(\bar{\partial}^{*}\right)^{-1} \mathcal{E} & 0
\end{array}\right)
$$

for some operators $\bar{\partial}^{-1}$ and $\left(\bar{\partial}^{*}\right)^{-1}$ inverting on the right the operators $\bar{\partial}$ and $\left(\bar{\partial}^{*}\right)^{-1}$ on an open manifold $\widetilde{M}$ which contains $M, \mathcal{E}$ is an extension operator extending $W^{k, q}(M)$ sections 
on $M$ to compactly supported sections in $W^{k, q}(\widetilde{M})$ (for $k \in\{0,1\}, q \in[1, \infty]$ ) and $\mathcal{R}$ is a restriction operator, restricting sections in $L^{q}(\widetilde{M})$ to sections in $L^{q}(M)$, see Section 2 of [1] for more details (where the notation for $M \subset \widetilde{M}$ was $M_{0} \subset M$ ).

Let $\psi$ be a real valued smooth Morse function on $\widetilde{M}$ and let

$$
\bar{\partial}_{\psi}^{-1}:=\mathcal{R} \bar{\partial}^{-1} e^{-\frac{2 i \psi}{h}} \mathcal{E}, \quad\left(\bar{\partial}_{\psi}^{*}\right)^{-1}:=\mathcal{R}\left(\bar{\partial}^{*}\right)^{-1} e^{2 i \psi / h} \mathcal{E}
$$

with notation as in (2.6). From [11, Lemma 2.2 and 2.3], we have following estimates

Lemma 2.3. For any $q>2$, there exists $\varepsilon>0$ and $C>0$ such that for all $\omega \in W^{1, q}\left(M, \underline{\mathbb{C}}^{n}\right)$, $\omega^{\prime} \in W^{1, q}\left(M, \underline{\mathbb{C}}^{n} \otimes\left(T^{0,1} M\right)^{*}\right)$, and $h>0$ small

$$
\left\|\left(\bar{\partial}_{\psi}^{*}\right)^{-1} \omega\right\|_{L^{2}} \leq C h^{\frac{1}{2}+\varepsilon}\|\omega\|_{W^{1, q}}, \quad\left\|\bar{\partial}_{\psi}^{-1} \omega^{\prime}\right\|_{L^{2}} \leq C h^{\frac{1}{2}+\varepsilon}\left\|\omega^{\prime}\right\|_{W^{1, q}} .
$$

Also, for $v \in W^{1, q}\left(M, \underline{\mathbb{C}}^{n}\right)$ and $v^{\prime} \in W^{1, q}\left(M, \underline{\mathbb{C}}^{n} \otimes\left(T^{0,1} M\right)^{*}\right)$ satisfying $\left.v\right|_{\partial M}=0$ and $\left.v^{\prime}\right|_{\partial M}=$ 0 , we have

$$
\begin{gathered}
\left\|\mathcal{E}^{*}\left(\bar{\partial}^{-1}\right)^{*} \mathcal{R}^{*}\left(e^{-2 i \psi / h} v\right)\right\|_{L^{2}} \leq C h^{\frac{1}{2}+\varepsilon}\|v\|_{W^{1, q}}, \\
\left\|\mathcal{E}^{*}\left(\left(\bar{\partial}^{*}\right)^{-1}\right)^{*} \mathcal{R}^{*}\left(e^{-2 i \psi / h} v^{\prime}\right)\right\|_{L^{2}} \leq C h^{\frac{1}{2}+\varepsilon}\left\|v^{\prime}\right\|_{W^{1, q}}
\end{gathered}
$$

Notice that, with

$$
D_{\psi}^{-1}=\left(\begin{array}{cc}
0 & \bar{\partial}_{\psi}^{-1} \\
\left(\bar{\partial}_{\psi}^{*}\right)^{-1} & 0
\end{array}\right)
$$

we have $D^{-1} V_{\psi}=D_{\psi}^{-1} V$. Hence applying $D^{-1}$ to both sides of (2.5) yields

and hence

$$
\left(\operatorname{Id}+D_{\psi}^{-1} V\right)\left(\begin{array}{c}
r_{h} \\
s_{h}
\end{array}\right)=-D_{\psi}^{-1} V\left(\begin{array}{l}
a \\
b
\end{array}\right)
$$

$$
\left\{\begin{array}{l}
r_{h}+\bar{\partial}_{\psi}^{-1}\left(Q^{-} s_{h}\right)=-\bar{\partial}_{\psi}^{-1}\left(Q^{-} b\right) \\
s_{h}+\left(\bar{\partial}_{\psi}^{*}\right)^{-1}\left(Q^{+} r_{h}\right)=-\left(\bar{\partial}_{\psi}^{*}\right)^{-1}\left(Q^{+} a\right)
\end{array}\right.
$$

Next we specialize to $a=0$ and find that $r_{h}$ satisfies

$$
\left(I-S_{h}\right) r_{h}=-\bar{\partial}_{\psi}^{-1}\left(Q^{-} b\right) \text { with } S_{h}:=\bar{\partial}_{\psi}^{-1} Q^{-} \bar{\partial}_{\psi}^{*-1} Q^{+}
$$

where $Q^{+}, Q^{-}$are viewed as multiplication operators. Similarly to Lemma 3.1 in [11], we have the following consequence of Lemma 2.3.

Lemma 2.4. Let $q>2$ and assume that $Q^{+} \in L^{\infty}\left(M, \operatorname{End}\left(\mathbb{C}^{n}\right)\right)$ and $Q^{-} \in W^{1, q}\left(M, \operatorname{End}\left(\mathbb{C}^{n}\right)\right)$, then $S_{h}$ is bounded on $L^{r}(M)$ for any $1<r \leq q$ and satisfies $\left\|S_{h}\right\|_{L^{r} \rightarrow L^{r}}=\mathcal{O}\left(h^{1 / r}\right)$ if $r>2$ and $\left\|S_{h}\right\|_{L^{2} \rightarrow L^{2}}=\mathcal{O}\left(h^{1 / 2-\varepsilon}\right)$ for any $0<\varepsilon<1 / 2$ small.

Using Lemma 2.4 equation (2.9) can be solved, for small enough $h$, through a Neumann series

$$
r_{h}:=-\sum_{j=0}^{\infty} S_{h}^{j} \bar{\partial}_{\psi}^{-1} Q^{-} b
$$

which defines an element of $L^{q}(M)$ for any $q \geq 2$. Substituting this expression for $r_{h}$ into equation (2.8) when $a=0$, we get that

$$
s_{h}=-\left(\bar{\partial}_{\psi}^{*}\right)^{-1} Q^{+} r_{h} .
$$


But using Lemma 2.4 and 2.3 for $s_{h}$ and $r_{h}$, we deduce that if $Q^{+} \in L^{\infty}\left(M\right.$, End $\left.\left(\mathbb{C}^{n}\right)\right)$ and $Q^{-} \in W^{1, q}\left(M, \operatorname{End}\left(\mathbb{C}^{n}\right)\right)$ for some $q>2$, then there exists $\varepsilon>0$ such that

$$
\left\|s_{h}\right\|_{L^{2}\left(M_{0}\right)}+\left\|r_{h}\right\|_{L^{2}\left(M_{0}\right)}=\mathcal{O}\left(h^{\frac{1}{2}+\varepsilon}\right) .
$$

Similarly, if one assumes that $Q^{-} \in L^{\infty}\left(M, \operatorname{End}\left(\mathbb{C}^{n}\right)\right)$ and $Q^{+} \in W^{1, q}\left(M, \operatorname{End}\left(\mathbb{C}^{n}\right)\right)$ for some $q>2$, then one can solve the system (2.8), with $b=0$, for any holomorphic $a$. In summary:

Proposition 2.1. Let $\Phi=\phi+i \psi$ be a Morse holomorphic function on $M$, and $b$ an antiholomorphic section of $\underline{\mathbb{C}}^{n} \otimes \Lambda^{0,1}(M)$. If $Q^{+} \in L^{\infty}\left(M, \operatorname{End}\left(\mathbb{C}^{n}\right)\right)$ and $Q^{-} \in W^{1, q}\left(M, \operatorname{End}\left(\mathbb{C}^{n}\right)\right)$ for some $q>2$, there exist solutions to $(D+V) F_{h}=0$ on $M$ of the form

$$
F_{h}=\left(\begin{array}{c}
e^{\Phi / h} r_{h} \\
e^{\bar{\Phi} / h}\left(b+s_{h}\right)
\end{array}\right)
$$

where $\left\|s_{h}\right\|_{L^{2}}+\left\|r_{h}\right\|_{L^{2}}=\mathcal{O}\left(h^{\frac{1}{2}+\varepsilon}\right)$ for some $\varepsilon>0$.

If instead $Q^{-} \in L^{\infty}\left(M, \operatorname{End}\left(\mathbb{C}^{n}\right)\right)$ and $Q^{+} \in W^{1, q}\left(M, \operatorname{End}\left(\mathbb{C}^{n}\right)\right)$ for some $q>2$, then for any holomorphic section of $\underline{\mathbb{C}}^{n}, a$, there exist solutions to $(D+V) G_{h}=0$ on $M$ of the form

$$
G_{h}=\left(\begin{array}{c}
e^{\Phi / h}\left(a+r_{h}\right) \\
e^{\bar{\Phi} / h} s_{h}
\end{array}\right)
$$

where $\left\|s_{h}\right\|_{L^{2}}+\left\|r_{h}\right\|_{L^{2}}=\mathcal{O}\left(h^{\frac{1}{2}+\varepsilon}\right)$ for some $\varepsilon>0$.

Note that for arbitrary $(a, b)$ satisfying (2.4) , one can just add the solutions for $(a, 0)$ and $(0, b)$.

2.2. Identifying the potential. As explained above, the CGO-type elements in the null space of $D+V$ with $V$ a diagonal potential, suffice to identify the potential on all of $M$.

Proposition 2.2. Let $D=\left(\begin{array}{cc}0 & \bar{\partial}^{*} \\ \bar{\partial} & 0\end{array}\right)$, let $V_{1}, V_{2}$ be two sections of $\operatorname{End}(E)$ with $A=A^{\prime}=0$ when written in the form (2.1), and satisfying the regularity assumption (2.2). If the Cauchy data spaces $\mathcal{C}_{D+V_{1}}$ and $\mathcal{C}_{D+V_{2}}$ agree, then $V_{1}=V_{2}$ on all of $M$.

Proof. Given the construction of CGO-type solutions above, the proof now follows that of [11, Thm. 3.3] (and hence also the argument of Bughkeim [7]), we repeat the main arguments for the reader's convenience. Let $\Phi$ be a Morse holomorphic function with a critical point at $z_{0}$. The existence of such a function for a dense set of points $z_{0}$ of $M_{0}$ is insured by Proposition 2.1. For any $b_{1}, b_{2}$ anti-holomorphic sections of $\underline{\mathbb{C}}^{n} \otimes\left(T^{1,0} M\right)^{*}$, we can find

$$
F_{h}^{1}:=\left(\begin{array}{c}
e^{\Phi / h} r_{h}^{1} \\
e^{\bar{\Phi} / h}\left(b_{1}+s_{h}^{1}\right)
\end{array}\right), \quad F_{h}^{2}:=\left(\begin{array}{c}
e^{-\Phi / h} r_{h}^{2} \\
e^{-\bar{\Phi} / h}\left(b_{2}+s_{h}^{2}\right)
\end{array}\right)
$$

solutions of $\left(D+V_{1}\right) F_{h}^{1}=0$ and $\left(D+V_{2}^{*}\right) F_{h}^{2}=0$ as in (2.12) , where $r_{h}^{j}, s_{h}^{j}$ are constructed in Proposition 2.1.

Since $\mathcal{C}_{D+V_{1}}=\mathcal{C}_{D+V_{2}}$, there exists an $F_{h}$ satisfying

$$
\left(D+V_{2}\right) F_{h}=0 \quad \text { and } \quad i_{\partial M}^{*} F_{h}=i_{\partial M}^{*} F_{h}^{1} .
$$


In particular, $\left(D+V_{2}\right)\left(F_{h}^{1}-F_{h}\right)=\left(V_{2}-V_{1}\right) F_{h}^{1}$ and $i_{\partial M}^{*}\left(F_{h}^{1}-F_{h}\right)=0$. We use Green's formula and the vanishing of $F_{h}^{1}-F_{h}$ on the boundary to get

$$
0=\int_{M}\left\langle\left(D+V_{2}\right)\left(F_{h}^{1}-F_{h}\right), F_{h}^{2}\right\rangle=\int_{M}\left\langle\left(V_{2}-V_{1}\right) F_{h}^{1}, F_{h}^{2}\right\rangle .
$$

where $\langle\cdot, \cdot\rangle$ denotes the Hermitian scalar product on $E$. If we denote $Q_{2}^{ \pm}-Q_{1}^{ \pm}$by $Q^{ \pm}$, we can rewrite this as

$$
0=\int_{M} e^{-2 i \frac{\psi}{h}}\left(\left\langle Q^{-} b_{1}, b_{2}\right\rangle+\left\langle Q^{-} b_{1}, s_{h}^{2}\right\rangle+\left\langle Q^{-} s_{h}^{1}, b_{2}\right\rangle+\left\langle Q^{-} s_{h}^{1}, s_{h}^{2}\right\rangle\right)+e^{2 i \frac{\psi}{h}}\left\langle Q^{+} r_{h}^{1}, r_{h}^{2}\right\rangle
$$

By Proposition 2.1, we always have

$$
\int_{M} e^{-2 i \frac{\psi}{h}}\left\langle Q^{-} s_{h}^{1}, s_{h}^{2}\right\rangle+e^{2 i \frac{\psi}{h}}\left\langle Q^{+} r_{h}^{1}, r_{h}^{2}\right\rangle=\mathcal{O}\left(h^{1+\varepsilon}\right)
$$

for some $\varepsilon>0$. Next we choose $b_{1}=\theta e_{j}$ and $b_{2}=\theta e_{k}$, where $\theta$ is an anti-holomorphic 1-form which vanishes at all critical points of $\Phi$ in $M$ except at the critical point $z_{0} \in M$ of $\Phi$ (and $e_{j}, e_{k}$ denote vectors in the canonical basis of $\left.\mathbb{C}^{n}\right)$. The existence of $\theta$ is guaranteed by the Riemann-Roch theorem (see Lemma 4.1 in [9]). We observe by using stationary phase that, as $h \rightarrow 0$,

$$
\int_{M} e^{-2 i \psi / h}\left\langle Q^{-} b_{1}, b_{2}\right\rangle=C_{z_{0}} h e^{-2 i \psi\left(z_{0}\right) / h}\left\langle Q^{-}\left(z_{0}\right) e_{j}, e_{k}\right\rangle\left|\theta\left(z_{0}\right)\right|^{2}+o(h)
$$

for some constant $C_{z_{0}} \neq 0$. To show this, one can decompose the integral, using a smooth cutoff function, near $z_{0}$ and far from $z_{0}$. The part localized near $z_{0}$ is simply obtained by stationary phase while the other part is $o(h)$, as can be seen by integrating by parts once (using $\partial_{z} e^{i \psi / h}=i e^{i \psi / h}\left(\partial_{z} \psi\right) / h$ ) to gain an $h$ factor and then applying Riemann-Lebesgue to show that the remaining oscillating integral goes to 0 as $h \rightarrow 0$. There are no boundary terms in the integration by parts since, by (2.3), $\left.Q^{-}\right|_{\partial M}=0$.

Let us now consider the term with $\left\langle Q^{-} b_{1}, s_{h}^{2}\right\rangle$ in (2.15): using (2.11) we can write this as

$$
\int_{M}\left\langle e^{-2 i \psi / h} Q^{-} b_{1}, s_{h}^{2}\right\rangle=\int_{M} e^{-2 i \psi / h}\left\langle\mathcal{E}^{*}\left(\bar{\partial}^{*-1}\right)^{*} \mathcal{R}^{*} e^{-2 i \psi / h} Q^{-} b_{1}, Q_{2}^{+} r_{h}^{2}\right\rangle .
$$

Since $\left.Q^{-}\right|_{\partial M}=0$, we may use (2.7) to deduce that $\left\|\mathcal{E}^{*}\left(\bar{\partial}^{*-1}\right)^{*} \mathcal{R}^{*} e^{2 i \psi / h} Q^{-} b_{1}\right\|_{L^{2}}=\mathcal{O}\left(h^{1 / 2+\varepsilon}\right)$ and thus combining with Proposition 2.1, we find that

$$
\int_{M}\left\langle e^{2 i \psi / h} Q^{-} b_{1}, s_{h}^{2}\right\rangle=\mathcal{O}\left(h^{1+\varepsilon}\right) .
$$

the same argument shows that the term involving $\left\langle Q^{-} s_{h}^{1}, b\right\rangle$ in (2.15) is $\mathcal{O}\left(h^{1+\varepsilon}\right)$. These last two estimates combined with (2.17) and (2.16) imply that $Q_{1}^{-}\left(z_{0}\right)=Q_{2}^{-}\left(z_{0}\right)$ by letting $h \rightarrow 0$. The same proof using the complex geometric optics solution $G_{h}$ of Proposition 2.1] gives $Q_{1}^{+}\left(z_{0}\right)=\widetilde{Q}_{2}^{+}\left(z_{0}\right)$.

2.3. Reduction to the diagonal case. Proposition 2.2 is the result we wanted when the potential is diagonal. We now show that one can always reduce to the diagonal case, and thereby establish the full result.

We first rewrite the operator $D+V$ as follows

$$
D+V=\left(\begin{array}{cc}
0 & \left(\bar{\partial}+A^{\prime}\right)^{*} \\
\bar{\partial}+A & 0
\end{array}\right)+\left(\begin{array}{cc}
Q^{+} & 0 \\
0 & Q^{-}
\end{array}\right)
$$


The operators $\bar{\partial}_{A}:=\bar{\partial}+A$ and $\bar{\partial}_{A^{\prime}}:=\bar{\partial}+A^{\prime}$ are CR operators on the trivial bundle $\underline{\mathbb{C}}^{n}=M \times \mathbb{C}^{n}$ over $M$, so by Lemma 1.2 they induce holomorphic structures on $\underline{\mathbb{C}}^{n}$ and by Proposition 1.1 there are holomorphic trivializations $F_{A}, F_{A^{\prime}} \in C^{1+r} \cap W^{1+s, p}\left(M, \operatorname{End}\left(\underline{\mathbb{C}}^{n}\right)\right)$ such that

$$
F_{A}^{-1} \bar{\partial} F_{A}=\bar{\partial}_{A}, \quad F_{A^{\prime}}^{-1} \bar{\partial} F_{A^{\prime}}=\bar{\partial}_{A^{\prime}} .
$$

From the factorization

$$
D+V=\left(\begin{array}{cc}
F_{A^{\prime}}^{*} & 0 \\
0 & F_{A}^{-1}
\end{array}\right)\left[\left(\begin{array}{cc}
0 & \bar{\partial}^{*} \\
\bar{\partial} & 0
\end{array}\right)+\left(\begin{array}{cc}
\left(F_{A^{\prime}}^{*}\right)^{-1} Q^{+} F_{A}^{-1} & 0 \\
0 & F_{A} Q^{-} F_{A^{\prime}}^{*}
\end{array}\right)\right]\left(\begin{array}{cc}
F_{A} & 0 \\
0 & \left(F_{A^{\prime}}^{*}\right)^{-1}
\end{array}\right)
$$

we see that the Cauchy data space of $D+V$ is determined by the Cauchy data space of $D+\widetilde{V}$ and the boundary values of $F_{A}, F_{A^{\prime}}$, where

$$
\widetilde{V}:=\left(\begin{array}{cc}
\left(F_{A^{\prime}}^{*}\right)^{-1} Q^{+} F_{A}^{-1} & 0 \\
0 & F_{A} Q^{-} F_{A^{\prime}}^{*}
\end{array}\right) .
$$

(Under assumption (2.2), one has $\widetilde{V} \in W^{1, q}\left(M, \operatorname{End}\left(\mathbb{C}^{n}\right)\right.$ ) for $q>2$.) We will show that, when the Cauchy data space of two Dirac-type operators coincide, it is possible to choose these isomorphisms consistently at the boundary.

Proposition 2.5. Let $A_{i}, Q_{i}^{ \pm}$satisfy the regularity assumption (2.2), for $i=1,2$. If $\left(\begin{array}{cc}Q_{1}^{+} & \left(\bar{\partial}+A_{1}^{\prime}\right)^{*} \\ \bar{\partial}+A_{1} & Q_{1}^{-}\end{array}\right)$and $\left(\begin{array}{cc}Q_{2}^{+} & \left(\bar{\partial}+A_{2}^{\prime}\right)^{*} \\ \bar{\partial}+A_{2} & Q_{2}^{-}\end{array}\right)$have the same Cauchy data at $\partial M$, then there exist bundle isomorphisms $\widetilde{F}_{A_{1}}, \widetilde{F}_{A_{1}^{\prime}}$ and $\widetilde{F}_{A_{2}}, \widetilde{F}_{A_{2}^{\prime}}$ as in (2.18) such that $\widetilde{F}_{A_{1}}=\widetilde{F}_{A_{2}}$ on $\partial M$ and $\widetilde{F}_{A_{1}^{\prime}}=\widetilde{F}_{A_{2}^{\prime}}$ on $\partial M$.

Proof. Let $F_{A_{1}}, F_{A_{1}^{\prime}}$ and $F_{A_{2}}, F_{A_{2}^{\prime}}$ be as in (2.18), we will show that one can modify $F_{A_{1}^{\prime}}$ and $F_{A_{2}^{\prime}}$ to satisfy the requirements of the proposition.

Our main tools will be the CGO-type solutions from $\$ 2.1$ and the following orthogonality condition for a function on $\partial M$ to extend holomorphically into the interior. The condition was derived in [11] and essentially is a basic computation using the Hodge decomposition. We refer the reader to Lemma 4.1 of [11] for a detailed proof.

Lemma 2.6. A complex valued function $f \in H^{1 / 2}(\partial M)$ is the restriction of a holomorphic function if and only if

$$
\int_{\partial M} f i_{\partial M}^{*} \eta=0
$$

for all 1-forms $\eta \in C^{\infty}\left(M ;\left(T^{1,0} M\right)^{*}\right)$ satisfying $\bar{\partial} \eta=0$.

Notice that, since $\partial: H^{1}(M) \rightarrow L^{2}\left(M ;\left(T^{1,0} M\right)^{*}\right)$ is surjective, the forms $\eta$ in the lemma can be replaced by $\partial \theta$ with $\theta$ a harmonic function.

Let $\Phi$ be a holomorphic Morse function and let $a$ and $b$ satisfy (2.4), applying Proposition 2.1 to the system $D+\widetilde{V_{1}}$, we can find $U_{h}^{1}$ of the form

$$
U_{h}^{1}=\left(\begin{array}{c}
e^{\Phi / h}\left(a+r_{h}^{1}\right) \\
e^{\bar{\Phi} / h}\left(s_{h}^{1}\right)
\end{array}\right)
$$

such that $\left(D+\widetilde{V_{1}}\right) U_{h}^{1}=0$. From (2.19) it follows that

$$
\widetilde{U}_{h}^{1}=\left(\begin{array}{c}
e^{\Phi / h} F_{A_{1}}^{-1}\left(a+r_{h}^{1}\right) \\
e^{\bar{\Phi} / h} F_{A_{1}^{\prime}}^{*}\left(s_{h}^{1}\right)
\end{array}\right)
$$


satisfies $\left(D+V_{1}\right) \widetilde{U}_{h}^{1}=0$.

Similarly, using the holomorphic Morse function $-\Phi$, and $b$ satisfying (2.4), we can find a solution to

$$
\left(\begin{array}{cc}
\left(Q_{2}^{+}\right)^{*} & \left(\bar{\partial}+A_{2}\right)^{*} \\
\bar{\partial}+A_{2}^{\prime} & \left(Q_{2}^{-}\right)^{*}
\end{array}\right) \widetilde{U}_{h}^{2}=0, \text { of the form } \widetilde{U}_{h}^{2}=\left(\begin{array}{c}
e^{-\Phi / h} F_{A_{2}^{\prime}}^{-1}\left(r_{h}^{2}\right) \\
e^{-\bar{\Phi} / h} F_{A_{2}}^{*}\left(b+s_{h}^{2}\right)
\end{array}\right) .
$$

Note that $\widetilde{U}_{h}^{2}$ is in the null space of $\left(D+V_{2}\right)^{*}$. Using the equality of the Cauchy data for $D+V_{1}$ and $D+V_{2}$, we can find an element $V_{h}$ in the null space of $D+V_{2}$ whose boundary data agrees with that of $\widetilde{U}_{h}^{1}$. Hence we obtain no boundary terms when we apply Green's formula to see

$$
\int_{M}\left\langle\left(D+V_{2}\right) \widetilde{U}_{h}^{1}, \widetilde{U}_{h}^{2}\right\rangle=\int_{M}\left\langle\left(D+V_{2}\right)\left(\widetilde{U}_{h}^{1}-V_{h}\right), \widetilde{U}_{h}^{2}\right\rangle=0 .
$$

Applying the remainder estimates from Proposition 2.1 we find that, as $h \rightarrow 0$,

$$
\begin{gathered}
0=\int_{M}\left\langle\left(D+V_{2}\right) \widetilde{U}_{h}^{1}, \widetilde{U}_{h}^{2}\right\rangle=\int_{M}\left\langle\left(V_{2}-V_{1}\right) \widetilde{U}_{h}^{1}, \widetilde{U}_{h}^{2}\right\rangle=\int_{M}\left\langle\left(\begin{array}{cc}
Q_{2}^{+}-Q_{1}^{+} & A_{2}^{\prime *}-A_{1}^{\prime *} \\
A_{2}-A_{1} & Q_{2}^{-}-Q_{1}^{-}
\end{array}\right) \widetilde{U}_{h}^{1}, \widetilde{U}_{h}^{2}\right\rangle \\
=\int_{M}\left\langle\left(A_{2}-A_{1}\right) F_{A_{1}}^{-1}\left(a+r_{h}^{1}\right), F_{A_{2}}^{*}\left(b+s_{h}^{2}\right)\right\rangle+o(1) \\
=\int_{M}\left\langle F_{A_{2}}\left(A_{2}-A_{1}\right) F_{A_{1}}^{-1} a, b\right\rangle+o(1)
\end{gathered}
$$

and hence

$$
\int_{M}\left\langle F_{A_{2}}\left(A_{2}-A_{1}\right) F_{A_{1}}^{-1} a, b\right\rangle=0
$$

for all $a$ and $b$ as above. Using the relations $\bar{\partial} F_{A_{j}}=F_{A_{j}} A_{j}$, this integral becomes

$$
0=\int_{M}\left\langle\bar{\partial}\left(F_{A_{2}} F_{A_{1}}^{-1} a\right), b\right\rangle=\int_{\partial M} i_{\partial M}^{*}\left\langle F_{A_{2}} F_{A_{1}}^{-1} a, b\right\rangle .
$$

We are free to choose the holomorphic section $a$ and the antiholomorphic 1-form $b$. Denoting $\left(e_{1}, \ldots, e_{n}\right)$ the canonical (holomorphic) basis of $\underline{\mathbb{C}}^{n}$, we choose $a=e_{k}$ and $b=\partial\left(\theta e_{j}\right)$ where $\theta$ is a harmonic function $\bar{\partial} \partial \theta=0$. Then if we denote by $\left(F_{A_{2}} F_{A_{1}}^{-1}\right)_{j, k}$ the $(j, k)$ component of the matrix $i_{\partial M}^{*} F_{A_{2}} F_{A_{1}}^{-1}$, we have, by Lemma 2.6, that each component of the endomorphism extends holomorphically into $M$ and we see that $i_{\partial M}^{*} F_{A_{2}} F_{A_{1}}^{-1}$ admits a holomorphic extension $F$.

This function $F$ is invertible. Indeed, switching the indices 1 and 2, we find a holomorphic extension function with boundary value $i_{\partial M}^{*} F_{A_{1}} F_{A_{2}}^{-1}$. The composition of these functions is holomorphic and equal to the identity on $\partial M$, hence on all of $M$.

Notice that the endomorphisms $\widetilde{F}_{A_{1}}=F_{A_{1}}, \widetilde{F}_{A_{2}}=F^{-1} F_{A_{2}}$ satisfy the requirements of Proposition 2.5, since

$$
\left.\widetilde{F}_{A_{2}}\right|_{\partial M}=\left.\left(F^{-1} F_{A_{2}}\right)\right|_{\partial M}=\left.F_{A_{1}}\right|_{\partial M}=\left.\widetilde{F}_{A_{1}}\right|_{\partial M}
$$

Using similar arguments, we can find a holomorphic extension $F^{\prime}$ of $F_{A_{1}^{\prime}} F_{A_{2}^{\prime}}^{-1}$ from the boundary of $M$, and then $\widetilde{F}_{A_{1}^{\prime}}=F_{A_{1}^{\prime}}, \widetilde{F}_{A_{2}^{\prime}}=\left(F^{\prime}\right)^{-1} F_{A_{2}^{\prime}}$ will satisfy the requirements of Proposition 2.5, thus completing the proof. 
To summarize, by Proposition 2.5 we are able to conjugate the operator $D+V_{j}$ into the operator $D+\widetilde{V}_{j}$ where $\widetilde{V}_{j}$ has block structure with only diagonal entries. Furthermore, since $\widetilde{F}_{A_{1}}=\widetilde{F}_{A_{2}}$ and $\widetilde{F}_{A_{1}^{\prime}}=\widetilde{F}_{A_{2}^{\prime}}$ along the boundary, we have that $\mathcal{C}_{D+\widetilde{V}_{1}}=\mathcal{C}_{D+\widetilde{V}_{2}}$. We can now apply Proposition 2.2 to $D+\widetilde{V}_{j}$ and prove

Theorem 2.7. Let $V_{1}, V_{2}$ be two sections of $\operatorname{End}(E)$ satisfying the regularity assumption $(\underline{2.2})$, and $D:=\left(\begin{array}{cc}0 & \bar{\partial}^{*} \\ \bar{\partial} & 0\end{array}\right)$. If the Cauchy data spaces $\mathcal{C}_{D+V_{1}}$ and $\mathcal{C}_{D+V_{2}}$ agree, then there exist $\mathrm{C}^{1}$ bundle isomorphisms $F, G$ of $\underline{\mathbb{C}}^{n}$ such that $\left.F\right|_{\partial M}=\left.G\right|_{\partial M}=\operatorname{Id}$ and, as operators,

$$
D+V_{2}=\left(\begin{array}{cc}
G & 0 \\
0 & F^{-1}
\end{array}\right)\left(D+V_{1}\right)\left(\begin{array}{cc}
F & 0 \\
0 & G^{-1}
\end{array}\right) .
$$

The proof of Theorem 2 is an immediate consequence of Theorem 2.7 and Lemma 1.3 ,

\section{THE SECOND ORDER CASE}

In this section, we use the result for Dirac-type systems to establish Theorem 1, The Cauchy data of a connection Laplacian plus potential on a surface with boundary determines the connection and the potential up to a gauge transformation equal to the identity at the boundary.

The connection between the second order system and the Dirac-type system is through the Bochner-Kodaria formula (1.3). Indeed, this formula shows that

$$
u \in C^{\infty}(M ; E) \text { satisfies }\left(\nabla^{*} \nabla+W\right) u=0
$$

is equivalent to

$$
\left(u, \bar{\partial}^{\nabla} u\right) \in C^{\infty}\left(M ; E \oplus\left(T_{0,1}^{*} \otimes E\right)\right) \text { satisfies }\left(\begin{array}{cc}
\Omega+W & \left(\bar{\partial}^{\nabla}\right)^{*} \\
\bar{\partial}^{\nabla} & - \text { Id }
\end{array}\right)\left(\begin{array}{c}
u \\
\bar{\partial}^{\nabla} u
\end{array}\right)=\left(\begin{array}{l}
0 \\
0
\end{array}\right) .
$$

Using the following proposition, we can readily apply the results above if we define $A$ by $\bar{\partial}^{\nabla}=\bar{\partial}+A$, and let

$$
D+V=\left(\begin{array}{cc}
\Omega+W & (\bar{\partial}+A)^{*} \\
\bar{\partial}+A & -\mathrm{Id}
\end{array}\right) .
$$

Proposition 3.1. The Cauchy data space of $\nabla^{*} \nabla+W$ determines the Cauchy data space of $D+V$.

Proof. Replacing $E$ with a trivial $\mathbb{C}^{n}$ bundle, we can write the connection as $\nabla=d+X$ for some one-form $X$. The boundary determination result of [11, Prop. 4.1] extends to show that the Cauchy data of $\nabla^{*} \nabla+W$ determines both $\left.X\right|_{\partial M}$ and $\left.W\right|_{\partial M}$.

Suppose that $L_{1}=\nabla_{1}^{*} \nabla_{1}+W_{1}$ and $L_{2}=\nabla_{2}^{*} \nabla_{2}+W_{2}$ have the same Cauchy data, and define $D+V_{1}$ and $D+V_{2}$ as above. Let $\left(\begin{array}{l}u \\ v\end{array}\right)$ satisfy $\left(D+V_{1}\right)\left(\begin{array}{l}u \\ v\end{array}\right)=\left(\begin{array}{l}0 \\ 0\end{array}\right)$ so that $u$ is in the null space of $L_{1}$ and $v=\left(\bar{\partial}+A_{1}\right) u$. By assumption there is an element $w$ in the null space of $\nabla_{2}^{*} \nabla_{2}+W_{2}$ such that

$$
\left.w\right|_{\partial M}=\left.u\right|_{\partial M},\left.\quad \nabla_{2}(\nu) w\right|_{\partial M}=\left.\nabla_{1}(\nu) u\right|_{\partial M}
$$

where $\nu$ represents a normal vector to the boundary. We will be done if we show that the element $\left(\begin{array}{c}w \\ \left(\bar{\partial}+A_{2}\right) w\end{array}\right)$ which is in the null space of $\left(D+V_{2}\right)$ has the same boundary values as $\left(\begin{array}{l}u \\ v\end{array}\right)$. However we already know that $\left.\nabla_{2} w\right|_{\partial M}=\left.\nabla_{1} u\right|_{\partial M}$ since the connection one-form restricted 
to the boundary is determined by the Cauchy space of $L_{j}$ and the normal derivatives coincide by assumption, and hence

$$
\left.\left(\bar{\partial}+A_{2}\right) w\right|_{\partial M}=\left.\left(\nabla_{2} w\right)^{1,0}\right|_{\partial M}=\left.\left(\nabla_{1} u\right)^{1,0}\right|_{\partial M}=\left.v\right|_{\partial M}
$$

as required.

Thus if $\mathcal{C}_{L_{1}}=\mathcal{C}_{L_{2}}$, we know from Theorem 2 that there exist bundle isomorphisms $F$ and $G$, equal to the identity at the boundary, such that

$$
\left(\begin{array}{cc}
\Omega_{1}+W_{1} & A_{1}^{*} \\
A_{1} & -\mathrm{Id}
\end{array}\right)=\left(\begin{array}{cc}
G & 0 \\
0 & F^{-1}
\end{array}\right)\left(\begin{array}{cc}
\Omega_{2}+W_{2} & A_{2}^{*} \\
A_{2} & -\mathrm{Id}
\end{array}\right)\left(\begin{array}{cc}
F & 0 \\
0 & G^{-1}
\end{array}\right)+\left(\begin{array}{cc}
0 & G \bar{\partial}^{*} G^{-1} \\
F^{-1} \bar{\partial} F & 0
\end{array}\right) .
$$

We underline the fact that the adjoints $A_{j}^{*}$ are adjoints of $A_{j}$ considered as maps from $E$ to $E \otimes\left(T^{(0,1)} M\right)^{*}$, where $E$ is equipped with Hermitian products $\langle\cdot, \cdot\rangle_{E}$ and $E \otimes\left(T^{(0,1)} M\right)^{*}$ is equipped with the Hermitian product

$$
\left\langle u_{1} \otimes v_{1}, u_{2} \otimes v_{2}\right\rangle:=\frac{1}{2 i}\left\langle u_{1}, u_{2}\right\rangle_{E} *\left(v_{1} \wedge \overline{v_{2}}\right) .
$$

where $*$ is the Hodge star operator. The matrix valued form $A_{j}$ can be written $\mathcal{A}_{j} \otimes u_{j}$ for some $u_{j} \in \Lambda^{0,1}(M)$ and $\mathcal{A}_{j} \in \operatorname{End}(E)$, we define the adjoint of $A_{j}$ as an element of $\operatorname{End}(E) \otimes\left(T^{0,1} M\right)^{*}$ to be $A_{j}^{*_{E}}:=\mathcal{A}_{j}^{*} \otimes \overline{u_{j}} \in \operatorname{End}(E) \otimes\left(T^{1,0} M\right)^{*}$ where $\mathcal{A}_{j}^{*}$ is simply the adjoint of $\mathcal{A}_{j}$ with respect to $\langle\cdot, \cdot\rangle_{E}$. Thus, one has $A_{j}^{*}=i * A_{j}^{*_{E}} \wedge$. Denoting $\nabla_{j}=d+X_{j}$, the fact that $\nabla_{j}$ is a Hermitian connection implies that $X_{j}=A_{j}-A_{j}^{* E}$. Now, (3.1) implies that

$$
F=G^{-1}, \quad A_{1}=F^{-1} A_{2} F+F^{-1} \bar{\partial} F, \quad A_{1}^{*}=G A_{2}^{*} G^{-1}+G \bar{\partial}^{*} G^{-1} .
$$

Therefore, using also that $\bar{\partial}^{*}=-i * \partial$, we deduce

$$
F^{-1} X_{2} F=F^{-1}\left(A_{2}-A_{2}^{* E}\right) F=A_{1}-A_{1}^{*_{E}}-F^{-1} \bar{\partial} F-F^{-1} \partial F=X_{1}-F^{-1} d F .
$$

This shows that $F^{-1} \Omega_{2} F=\Omega_{1}$ since $\Omega_{j}$ is the curvature of $\nabla_{j}$, but using again (3.1) one also get $F^{-1} W_{2} F=W_{1}$. By (3.2), we also obtain

$$
\bar{\partial} F^{-1}+\left(A_{1} F^{-1}-F^{-1} A_{2}\right)=0, \quad \bar{\partial} F^{*}+\left(A_{1} F^{*}-F^{*} A_{2}\right)=0
$$

with $\left.F^{-1}\right|_{\partial M}=\left.F^{*}\right|_{\partial M}=\mathrm{Id}$, and thus by uniqueness of the solution of the elliptic boundary value problem

we deduce $F^{-1}=F^{*}$. We thus have proved

$$
\bar{\partial} H+A_{1} H-H A_{2}=0,\left.\quad H\right|_{\partial M}=\mathrm{Id}
$$

Theorem 3.1. If the two operators $L_{1}, L_{2}$ have same Cauchy data space, then there exists a unitary bundle isomorphism $F: E \rightarrow E$ such that $F \nabla_{1} F^{-1}=\nabla_{2}, F W_{1} F^{-1}=W_{2}$ and $\left.F\right|_{\partial M}=$ Id.

\section{Systems in DOMAins OF $\mathbb{C}$}

In our subsequent applications we will need to consider $\partial$-type systems where the leading symbols are not self-adjoint operators. We consider in this section such first order systems in a domain $\Omega \subset \mathbb{C}$. Let $D$ be the operator acting on $H^{1}\left(\Omega, \mathbb{C}^{m} \oplus \mathbb{C}^{n}\right)$, by

$$
D=\left(\begin{array}{cc}
\bar{\partial} & 0 \\
0 & \partial
\end{array}\right), \text { with } \bar{\partial}\left(\begin{array}{c}
u_{1} \\
\ldots \\
u_{m}
\end{array}\right)=\left(\begin{array}{c}
\partial_{\bar{z}} u_{1} \\
\ldots \\
\partial_{\bar{z}} u_{m}
\end{array}\right), \partial\left(\begin{array}{c}
v_{1} \\
\ldots \\
v_{n}
\end{array}\right)=\left(\begin{array}{c}
\partial_{z} v_{1} \\
\ldots \\
\partial_{z} v_{n}
\end{array}\right)
$$


where $\partial_{z}:=\partial_{x}-i \partial_{y}$ and $\partial_{\bar{z}}:=\partial_{x}+i \partial_{y}$ in the $z=x+i y$ complex coordinate on $\Omega$. We consider a potential $V \in \operatorname{End}\left(\mathbb{C}^{m} \oplus \mathbb{C}^{n}\right)$ satisfying $V=\left(\begin{array}{cc}A & Q^{+} \\ Q^{-} & B\end{array}\right)$ with

$$
\begin{gathered}
Q^{+} \in W^{1, q}\left(\Omega ; M_{n \times m}(\mathbb{C})\right), Q^{+} \in W^{1, q}\left(\Omega ; M_{m \times n}(\mathbb{C})\right), q>2 \\
\qquad A \in C^{r} \cap W^{s, p}\left(\Omega ; M_{m \times m}(\mathbb{C})\right), B \in C^{r^{\prime}} \cap W^{s^{\prime}, p^{\prime}}\left(\Omega ; M_{n \times n}(\mathbb{C})\right), \\
\text { with } 0<r<s, \quad p \in(1, \infty) \text { satisfy } r+s>1, \quad r \notin \mathbb{N}, \quad s p>2 m+2 \\
\text { and } 0<r^{\prime}<s^{\prime}, \quad p \in(1, \infty) \text { satisfy } r^{\prime}+s^{\prime}>1, \quad r^{\prime} \notin \mathbb{N}, \quad s^{\prime} p^{\prime}>2 n+2
\end{gathered}
$$

where $M_{m \times n}(\mathbb{C})$ denote the set of complex valued $m \times n$ matrices. We define the Cauchy data of the $D+V$ system by

$$
\mathcal{C}_{D+V}:=\left\{\left(\begin{array}{l}
\left.u\right|_{\partial \Omega} \\
\left.v\right|_{\partial \Omega}
\end{array}\right) ;\left(\begin{array}{l}
u \\
v
\end{array}\right) \in H^{1}\left(\Omega, \mathbb{C}^{m} \oplus \mathbb{C}^{n}\right) \mid(D+V)\left(\begin{array}{l}
u \\
v
\end{array}\right)=0\right\}
$$

The proofs of Proposition 2.5 and Proposition 2.2 easily extend to cover this situation:

Theorem 4.1. Let $V_{j}=\left(\begin{array}{cc}A_{j} & Q_{j}^{+} \\ Q_{j}^{-} & B_{j}\end{array}\right)$ be matrix valued potentials satisfying regularity conditions in [4.1). If $\mathcal{C}_{D+V_{1}}=\mathcal{C}_{D+V_{2}}$, then there exists invertible matrices $F_{j} \in C^{1}\left(\Omega, \operatorname{End}\left(\mathbb{C}^{m}\right)\right)$ and $G_{j} \in C^{1}\left(\Omega, \operatorname{End}\left(\mathbb{C}^{n}\right)\right)$ such that $F_{1}=F_{2}, G_{1}=G_{2}$ on $\partial \Omega$ and

$$
\bar{\partial} F_{j}=F_{j} A_{j}, \quad \partial G_{j}=G_{j} B_{j} .
$$

Furthermore, $Q_{1}^{+}=F Q_{2}^{+} G^{-1}$ and $Q_{1}^{-}=G Q_{2}^{-} F^{-1}$ where

$$
F:=F_{1}^{-1} F_{2}, \quad G=G_{1}^{-1} G_{2} .
$$

\section{REFERENCES}

[1] K. Astala, L. Päivärinta, Calderón's inverse conductivity problem in the plane, Ann. of Math. (2) 163 (2006), no. 1, 265-299.

[2] K. Astala, M. Lassas, L. Päivärinta, Calderón's inverse problem for anisotropic conductivity in the plane, Comm. Partial Differential Equations 30 (2005), no. 1-3, 207-224.

[3] R. Beals and R. Coifman, The spectral problem for Davey-Stewarson and the Ishimory hierarchies, Nonlinear evolution equations: Integrability and spectral methods, pages 15-23. Manchester University Press, 1988.

[4] M.I. Belishev, The Calderon problem for two dimensional manifolds by the BC-method, SIAM J. Math. Anal. 35, no 1, (2003), 172-182.

[5] N. Berline, E. Getzler, M. Vergne Heat kernels and Dirac operators. Corrected reprint of the 1992 original. Grundlehren Text Editions. Springer-Verlag, Berlin, 2004. x+363 pp.

[6] R. Brown, G. Uhlmann, Uniqueness in the inverse conductivity problem with less regular conductivities, Comm. PDE 22 (1997), 1009-1027.

[7] A.L. Bukhgeim, Recovering a potential from Cauchy data in the two-dimensional case. J. Inverse Ill-Posed Probl. 16 (2008), no. 1, 19-33.

[8] O. Forster, Lectures on Riemann surfaces, GTM 81, Springer.

[9] C. Guillarmou, L. Tzou, Calderón inverse problem for Schrödinger operator on Riemann surfaces. Proceedings of the Centre for Mathematics and its Applications, Vol 44 (2010) - proceedings of the AMSI-ANU Workshop on Spectral Theory and Harmonic Analysis.

[10] C. Guillarmou, L. Tzou, Calderón inverse Problem with partial data on Riemann Surfaces, Duke Math. J. 158 (2011), no. 1, 83-120.

[11] C. Guillarmou, L. Tzou, Identification of a connection from Cauchy data space on a Riemann surface with boundary, GAFA 21, no. 2, 393-418.

[12] G. Henkin, V. Michel, On the explicit reconstruction of a Riemann surface from its Dirichlet-Neumann operator, GAFA 17 (2007), 116-155. 
[13] G. Henkin, V. Michel, Inverse conductivity problem on Riemann surfaces. J. Geom. Anal. 18 (2008), no. 4, 1033-1052.

[14] G. Henkin, R.G. Novikov, On the reconstruction of conductivity of bordered two-dimensional surface in $\mathbb{R}^{3}$ from electrical currents measurements on its boundary, arXiv:1003.4897. To appear in J. Geom. Anal.

[15] G. Henkin, M Santacesaria, Gel'fand-Calderón's inverse problem for anisotropic conductivities on bordered surfaces in $\mathbb{R}^{3}$, Inverse Problems 26 (2010), no. 9, 095011, 18 pp.

[16] C.D. Hill, M. Taylor Integrability of rough almost complex structures Journal of Geometric Analysis 13 (2003), No 1, 163-172.

[17] O.Y. Imanuvilov, G. Uhlmann, M. Yamamoto, Global uniqueness from partial Cauchy data in two dimensions, J. Amer. Math. Soc. 23 (2010), 655-691.

[18] O. Imanuvilov, G. Uhlmann, M. Yamamoto, Partial Cauchy data for general second order operators in two dimensions., arXiv:1010.5791

[19] O. Imanuvilov, G. Uhlmann and M. Yamamoto, On determination of second order operators from partial Cauchy data, Proceedings National Academy of Sciences, 108 (2011), 467-472.

[20] O. Imanuvilov, G. Uhlmann, M. Yamamoto, Inverse boundary value problem by measuring Dirichlet data and Neumann data on disjoint sets, preprint.

[21] H. Kang, G. Uhlmann, Inverse problem for the Pauli Hamiltonian in two dimensions, J. Fourier Anal. Appl. 10 (2004), no. 2, 201-215

[22] S. Kobayashi, Differential geometry of complex vector bundles. Publications of the Mathematical Society of Japan, 15. Kan Memorial Lectures, 5. Princeton University Press, Princeton, NJ; Iwanami Shoten, Tokyo, 1987. xii+305 pp.

[23] M. Lassas, G. Uhlmann, On determining a Riemannian manifold from the Dirichlet-to-Neumann map. Ann. Sci. École Norm. Sup. (4) 34 (2001), no. 5, 771-787.

[24] X. Li, Inverse scattering problem for the Schroedinger operator with external Yang-Mills potentials in two dimensions at fixed energy, Comm. Partial Differential Equations 30 (2005), no. 4-6, 451-482.

[25] D. McDuff, D. Salamon, J-holomorphic curves and symplectic topology. American Mathematical Society Colloquium Publications, 52. American Mathematical Society, Providence, RI, 2004. xii+669 pp.

[26] A. Nachman, Global uniqueness for a two dimensional inverse boundary value problem, Annals of Math 143 (1996), 7196.

[27] A. Newlander, L. Nirenberg, Complex coordinates in almost complex manifolds, Ann. of Math., 65 (1957), 391-404.

[28] R.G. Novikov, M. Santacesaria Global uniqueness and reconstruction for the multi-channel Gel'fandCalderón inverse problem in two dimensions. ArXiv 1012.4667.

[29] R. Schrader, M. Taylor Small h asymptotics for quantum partition functions associated to particles in external Yang-Mills potentials, Comm. Math. Phys. 92 (1984), 555-594.

[30] J. Sylvester, An anisotropic inverse boundary value problem Commun. Pure Appl. Math. 43 (1990) 20132

Institut de Mathématiques de Jussieu, U.M.R. 7586 CNRS, Université Paris 7, 175 RUE DU Chevaleret, 75013 PARis

E-mail address: albin@math.jussieu.fr

Département de Mathématiques et Applications, U.M.R. 8553 CNRS, Ecole Normale Supérieure, 45 Rue D'Ulm, F 75230 Paris, Cedex 05, France

E-mail address: cguillar@dma.ens.fr

Department of Mathematics,, The University of Arizona, 617 N. Santa Rita Ave. P.O. Box 210089., TuCsOn, AZ 857210089 USA

E-mail address: leo.tzou@gmail.com

University of California,, Irvine, 340 Rowland Hall, Irvine, CA 92697-3875, and Department of Mathematics, The University of Washington, C-449 Padelford Hall, Box 354350, Seattle, WASHINGTON 98195-4350 USA

E-mail address: gunther@math. washington.edu 